\title{
Primary Intrapelvic hydatid Gyst Presenting with Urinary Retention
}

Kanchan Dhungel, Kaleem Ahmad, Panna Lal Sah, Mukesh Kumar Gupta, RK Rauniyar, Sajid Ansari, Shailesh Adhikary', Vikal Chandra Shakya'.

Department of Radiodiagnosis \& Imaging and Department of Surgery', B.P. Koirala Institute of Health Sciences, Dharan, Nepal.

\section{Abstract:}

Although hydatid disease can affect any part of the body, hydatid cysts located at unusual sites may create diagnostic confusion. The primary involvement of the pelvic cavity is a very rare and patients usually present with pressure symptoms affecting the adjacent organs. Herein, we report a case of pelvic hydatid cyst describing the ultrasonography (USG) and computed tomography (CT) findings of disease.

Key words: Echinococcus granulosus, Hydatid cyst, Ultrasonography, Computed tomography.

\section{Introduction}

Hydatid cyst ( $\mathrm{HC})$, a parasitic infection caused by Echinococcus granulosus is a considerable medical and public health problem. It may develop in any part of the body, liver being the most common site followed by the lung [1]. Hydatid cysts located in the peritoneal cavity or pelvis is usually secondary to spontaneous rupture from a primary liver focus or surgical inoculation. Primary peritoneal hydatidosis is rare and has been reported to occur in only 2 percent of all abdominal hydatid disease cases [2]. Unusual location of this disease can cause diagnostic problem and serious complications. Although diagnosing hydatid disease at unusual location may be challenging hydatid cyst should always be considered in the differential diagnoses of the abdomino-pelvic masses in endemic regions of the world. The characteristic imaging findings such as cyst wall calcification, daughter cysts, or membrane detachment have been described in literature [3]. The radiologic signs are often nonspecific and reliability of serological is also not $100 \%$. The diagnosis is made through the combined assessment of clinical, radiological, and laboratory findings.

\section{Case Report}

A twenty year-old girl presented with a history of decrease flow of urine and sensation of incomplete emptying. On abdominal examination, tender firm mass was palpable in pelvis. Laboratory investigations revealed eosinophilia with normal radiological examination of chest. Ultrasonography of the abdomen revealed a large multicystic mass, measuring $10.7 \times 5.6 \mathrm{~cm}$, located posterior to the uterus with no visualization of ovaries and free intraperitoneal fluid [Fig. 1]. A subsequent CT scan demonstrated a large complex cyst in pelvis containing numerous smaller cysts giving a honeycomb appearance compressing the uterus anteriorly without any solid component [Fig. 2a and 2b]. The sonographic and CT findings were consistent with hydatid cyst. Although multiseptated daughter cysts may be mistaken for other cystic neoplasm of the ovary the presence of a thick, 
bilayered wall was suggestive of hydatid cyst. In view of cyst being located in the pelvis in a female patient with nonvisualization of ovaries, ovarian cystadenoma was provided as a differential diagnosis. There was no evidence of hydatid cyst elsewhere in the abdominal cavity. An indirect hemagglutination test for echinococcus granulosus was positive. At laparotomy, the cystic mass was posterior to the uterus with some adhesions with the uterus and rectum [Fig. 3]. Both fallopian tubes and ovaries appeared normal. The cyst was successfully excised in toto. Gross pathologic examination revealed a thick walled cyst with multiple internal cysts with translucent fluid. Histological examination confirmed the diagnosis of a hydatid cyst. Patient had uneventful recovery. There was no recurrence at follow up USG after 6 months.

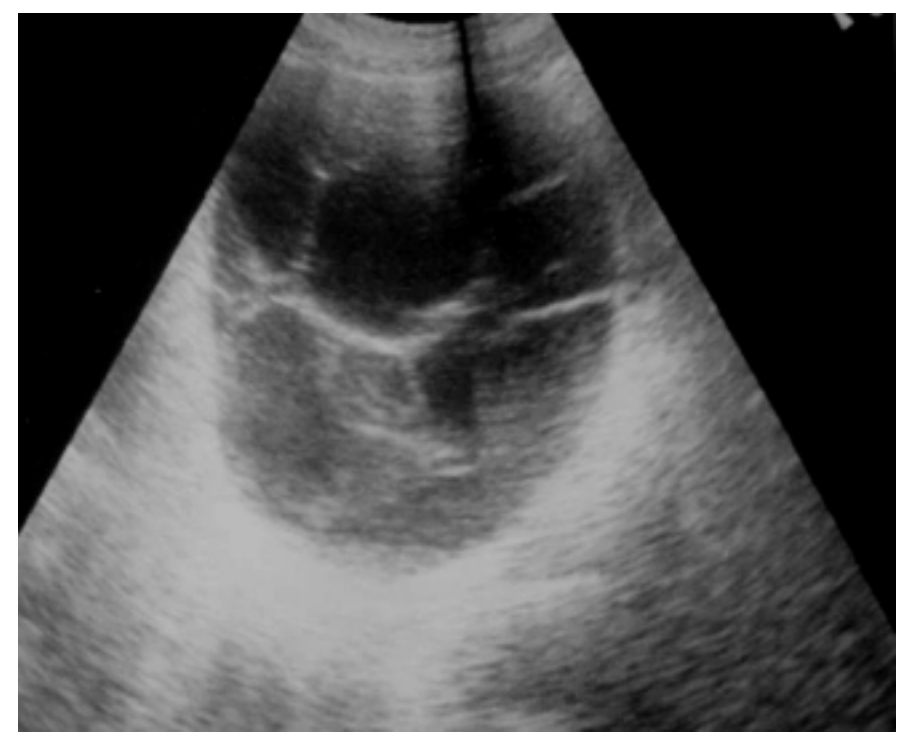

Fig.1: USG of pelvis showing a large cyst containing multiple daughter cysts posterior to the uterus.
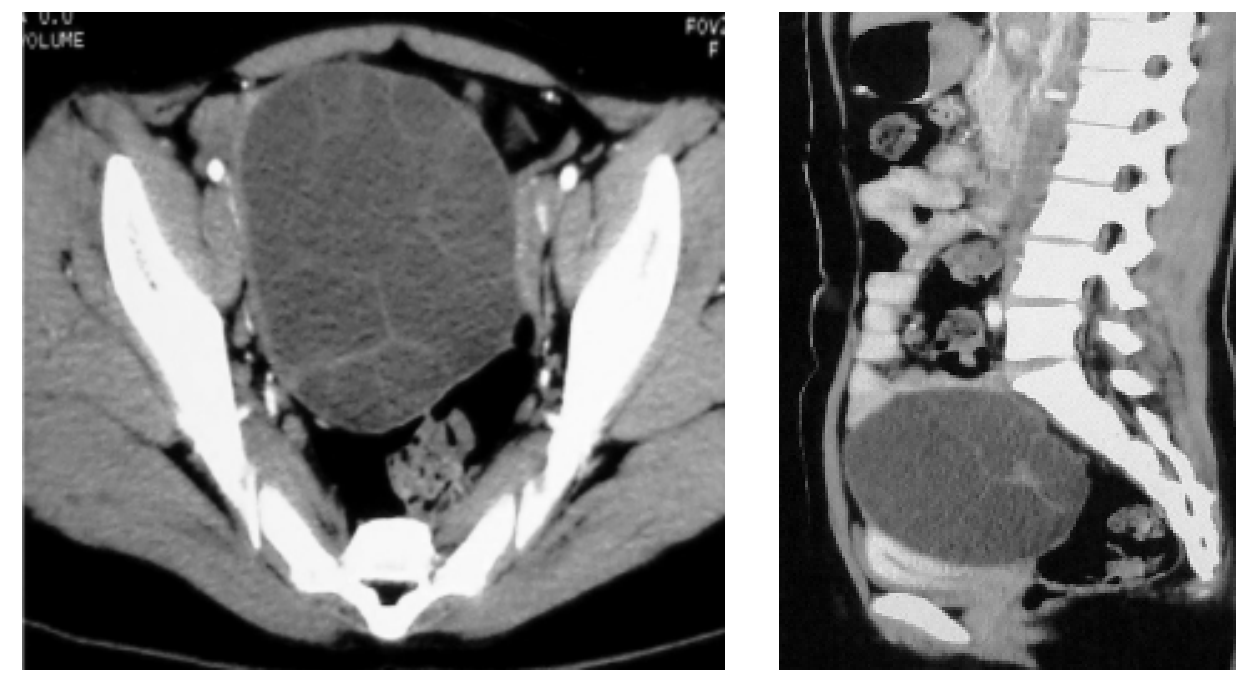

Fig.2: Contrast enhanced axial (Figure 2a) and sagittal (Figure 2b) CT images of the pelvis showing a large hydatid cyst with multiple daughter cysts posterior to the uterus compressing the uterus anteriorly. 


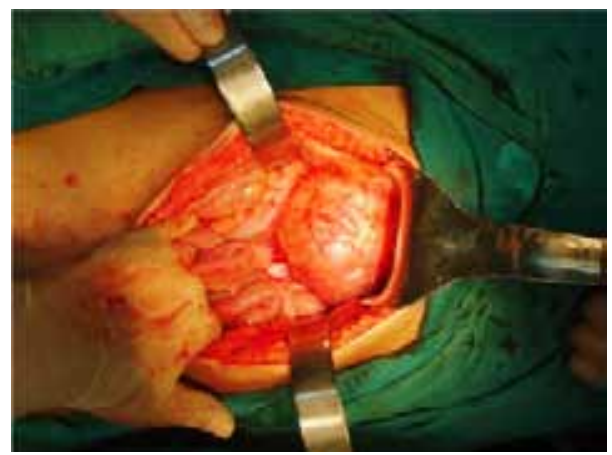

Fig.3: Intra-operative picture of the pelvic hydatid cyst.

\section{Discussion}

Hydatid disease (HD) is a parasitic infestation caused by Echinococcus granulosus and rarely by Echinococcus multilocularis. Once ingested eggs are hatched, they enter the portal circulation, get trapped in the liver [4] and enter the systemic circulation if they escape the hepatic filter and settle in the lungs or other organs and eventually develop into a cyst. The liver (59-75\%) is the most common organ affected, followed in by lung $(27 \%)$, kidney $(3 \%)$, bone (1-4\%), and brain (1-2\%). Other sites such as the heart, spleen, pancreas, omentum, ovaries, parametrium, pelvis, thyroid, orbit, retroperitoneum, and muscles are affected very rarely [5].

Peritoneal hydatid cyst, either primary or secondary, represents an uncommon disease. A cyst in the pelvic cavity can be considered primary only when no hydatid cysts are present at other sites in the body. The young girl in our case had the cyst primarily in the pelvis and no other hydatid cyst was detected in other organ or peritoneal cavity.

Symptoms of hydatid disease may be either due to local pressure caused by the cyst or systemic allergic reactions $[6,7]$. Symptoms depend on the size and location of the cyst. Pelvic hydatid cysts usually present as a nonspecific mass with pressure effects on urinary bladder and rectum [8]. Patients may present with pain, frequency, urinary retention and pain on micturition, constipation, weight loss and renal insufficiency. Our case presented with decrease flow of urine and sensation of incomplete emptying.

Diagnosis of the hydatid cyst is mainly on the basis of serologic tests and imaging techniques including USG, CT and MRI. Imaging may help to determine the accurate location and morphology of a cystic pelvic mass, and also exclude hydatid disease elsewhere.

The appearance of hydatid disease varies. Imaging findings range from purely cystic lesions to solid-appearing masses. A unilocular (type 1) cyst may mimic a simple cyst and multiseptated daughter cysts (type 2) may be mistaken for complex cyst [9]. However, the presence of a thick, bilayered wall is suggestive of hydatid disease. "Floating membranes", the detached endocyst inside the cavity, is highly specific for hydatid disease. Complete detachment of the membranes inside the cyst has been referred to as the "water lily sign" on sonography. Multivesicular cysts manifest as well-defined fluid collections in a honeycomb pattern, with multiple septa representing the walls of the daughter cysts. Our case had similar appearance. When daughter cysts are 
separated by the hydatid matrix (a material with mixed echogenicity), they show a "wheel spoke" pattern. The membrane from the endocyst may detach and precipitate to the bottom of the hydatid fluid to become hydatid sand. Mural nodularity suggests scolices [10]. Type 3 cysts appear as a bright echogenic focus with strong posterior acoustic shadowing. Ring-like or total calcification can be seen during the natural evolution which is commonly seen in the liver, spleen, and kidney.

On CT scan, daughter cysts usually have lower attenuation than that of the maternal matrix, and both the wall and internal septa often show contrast enhancement [9]. MRI shows the characteristic low signal intensity rim on T2-weighted images, representing the pericyst, which is rich in collagen [11]. Ultrasonography is the most sensitive imaging modality for the detection of membranes, septa, and hydatid sand within the cyst whereas CT is best in showing cyst wall calcification, cyst infection, and peritoneal seeding.

The combinations of radiologic and serologic tests especially in patients living in the endemic areas contribute to the diagnosis. However, surgical exploration may be necessary for definitive diagnosis.

\section{Conclusion}

This case has been presented to increase the awareness about this rare entity, to highlight the importance of recognizing the typical findings of hydatid cyst in cases undergoing evaluation of pelvic cysts, and the role of the imaging in its diagnosis. Hydatid disease should be considered in the differential diagnosis of unusual cystic swellings in the pelvis, particularly in a patient who has lived in an endemic area.

\section{References}

1. Mushtaque $\mathrm{M}$, Mir MF, Lone $\mathrm{ML}$, Batt SH. Solitary subcutaneous gluteal hydatid cyst: a case report. Eastern Journal of Medicine. 2010;15:76-79.

2. Parray FQ, Gagloo MA, Bhat AH, Chowdri NA, Noor MM. Peritoneal hydatidosis. The Internet Journal of Surgery. 2007;9(2).

3. Pedrosa I, Saiz A, Arrazola J, Ferreiros J, Pedrosa CS. Hydatid disease: radiologic and pathologic features and complications. Radiographics. 2000;20(3):795-817.

4. Hamamci EO, Besim $\mathrm{H}$, Korkmaz A. Unusual locations of hydatid disease and surgical approach. ANZ J Surg. $2004 ; 74: 356-360$.

5. Yuksel M, Demirpolat $G$, Sever A, Bakaris $S$, Bulbuloglu E, Elmas N. Hydatid disease involving some rare locations in the body: a pictorial essay. The Korean Journal of Radiology. 2007;8(6):531-540.

6. Akhan O, Ozmen MN, Dincer A, Sayek I, Gocmen A. Liver hydatid disease: long term results of percutaneous treatment. Radiology. 1996;198:259-264.

7. Beggs I. The radiology of hydatid disease. AJR Am J Roentgenol.1985; 145:639-648.

8. Seenu V, Misra MC, Tiwari SC, Jain R, Chandrashekhar C. Primary pelvic hydatid cyst presenting with obstructive uropathy and renal failure. Postgraduate Medical Journal. 1994;70:930-932.

9. Ishimitsu DN, Saouaf R, Kallman C, Balzer BL. Renal Hydatid Disease Radiographics. 2010;30:334-333.

10. King DJ. Ultasonography of echinococcal cysts. J clinical ultrasound. 1976;1 15:742-744.

1 1. G. Heirwegh, B. Claikens. Type I Hydatid cyst of the liver: Typical MRI features. JBR-BTR. 2005;88:136-137. 